\title{
Causality modelling of simulated temporally indexed events to construct a combat narrative
}

\author{
J.K.R. Tiong ${ }^{a}$ (iD) and B.A. Chiera ${ }^{a}$ (i) \\ ${ }^{a}$ UniSA STEM, University of South Australia, Adelaide, South Australia, 5000 \\ Email: junia.tiong@unisa.edu.au
}

\begin{abstract}
Combat simulation studies are commonly used to support decision-making on the basis of competing outputs, simulated under comparable conditions, for a selection of input design points. Such simulators have long been in use to support analysis in force design, operational requirements, mission area analysis and force-on-force analysis, necessitating high-resolution, closed-form and stochastic simulation capabilities. Each simulation typically runs as a black-box, making it difficult to discern the intermediate stochastically-varying series of events that form part of the combat narrative, detailing the sequence of events that led from the simulator inputs to the observed outputs.
\end{abstract}

Although the simulator runs in black-box mode, internal simulator data on stochastically-varying temporally indexed events over the combat spatial region are also captured. These events record an ordered series of events that, although stochastically seeded, reflect the fundamental logic of cause and effect. Understanding these event series through appropriate statistical modelling has the potential to provide key insights into the combat mission narrative and support decision-making around combat missions.

In this paper we present a first step towards constructing a combat mission narrative to support decision-making via a case study of internal combat simulation event series and their associated outputs. Statistical modelling of the internal data is challenged by the underlying simulator mechanisms - the use of Common Random Numbers (CRN) to reduce the variability in simulation output. CRN simulators are fraught with difficulties as they violate key statistical assumptions by design, requiring alternative, robust methods of analysis.

We adopt the use of the statistically robust Event Coincidence Analysis (ECA) to capture causality between events by providing a framework for quantifying the strength, directionality and time lag between two event series. The use of ECA is novel in this area of application; ECA has been recently adopted in the literature, predominantly in the areas of ecology, environment and health and is relatively under-explored in Defence, with current areas of application including armed conflict and hate-speech triggered terrorism.

An attractive feature of ECA is that it allows for significance testing of causality between two series, based on stochastic point processes with a prescribed inter-event time distribution and other higher-order properties, thus providing a differentiation between coincidence and causal events. Specifically, ECA considers two types of causal behaviour - precursor and trigger - with the former describing a series of events that typically occur before a secondary event takes place (mediated cause-and-effect) whereas the latter captures the concept of direct cause-and-effect between two events. Extensions of ECA include aggregation and conditionalisation; the former providing an integrated measure for coincidences that occur between several pairs of event series subject to some meaningful grouping mechanism and the latter allowing for the flexibility of interlinking multiple causal event series, to allow for conditioning of events on specific situations.

The R library coincalc was used to implement ECA as part of constructing a combat narrative. The case study provided four event series of interest - Movement, Detections, Shots and Kills - yielding combat narratives around the progression of Movement leading to Detection, being Shot and a Kill. Suggested combat narratives arising from the analyses conducted herein were: (i) standing can act as either a precursor or trigger to being identified or recognised while detection is a mediating, not direct, trigger for being shot. These results were supported by two different ECA methodologies; and (ii) for those simulations resulting in combat mission failure, precursory behaviour led to the failure of the overall mission over a relatively short time window, rather than a single, or series of, direct triggers.

Keywords: Combat simulation, temporally indexed event series, event coincidence analysis, precursor and trigger behaviours, combat narrative, decision-making 


\section{INTRODUCTION}

Simulation studies are commonly used to support decision-making on the basis of competing outputs, simulated under comparable conditions, for a selection of input design points. Combat simulators have long been in use to support analysis in force design, operational requirements, mission area analysis and force-on-force analysis, necessitating high-resolution, closed-form and stochastic simulation capabilities. These simulators typically run as a black-box, making it difficult to discern the intermediate stochastically-varying series of events between any given input and output leading to an outcome of interest. However understanding the interplay between simulator input, internal stochastic simulator behaviour and the simulated outputs is important to support insight into simulated combat mission narrative for decision-making and related activities.

Although the simulator runs as a black-box, internal data on stochastically-varying temporally indexed events over the combat spatial region are captured, with these events contributing to simulation output. These events have the potential to contribute to the narrative of how the combat mission unfolded by providing an inhomogeneous series of events occurring in space and time. Moreover, although the simulator is stochastically varying, these simulated temporally indexed events are designed to imitate reality; that is they are not intended to unfold randomly, but rather as an ordered series of events connected by cause and effect. Thus statistical modelling and understanding of such a narrative could provide critical information as part of overall combat mission progression to aid decision-making. A complication arises however, in that key to the simulator structure is the use of Common Random Numbers (CRN) to reduce the variability in simulation output (Heikes, et al., 1976). CRN simulators are fraught with difficulties as they violate key statistical assumptions by design, requiring alternative methods of analysis (Law, 2015).

In this paper we present a first step towards constructing a combat narrative in support of decision-making via a case study explored using the statistically robust Event Coincidence Analysis (ECA) (Donges, et al., 2016). ECA considers two types of causal behaviour - precursor and trigger - with the former describing a series of events that typically occur before a secondary event takes place (mediated cause-and-effect) whereas the latter captures the concept of direct cause-and-effect between two events. The use of ECA is novel in this area of application; ECA has been recently adopted in the literature, predominantly in the areas of ecology, environment and health (Templ, et al., 2021; Wiedermann, et al., 2021; Wolf, et al., 2021; Fdez-Arroyabe, et al., 2020) and is relatively under-explored in Defence, with current areas of application including armed conflict and hate-speech triggered terrorism (Scharwaechter \& Mueller, 2020; Schleussner, et al., 2016).

This paper is outlined as follows. In Section 2 we introduce the case study by exploring the characteristics of the temporally indexed event series under consideration before introducing ECA in Section 3. Section 4 focuses on constructing combat narratives for the case study using ECA on four stochastically-varying intermediate event series. Finally in Section 5 we give our conclusions and future directions.

\section{CASE STUDY: TEMPORALLY INDEXED EVENT SERIES OF A COMBAT SIMULATOR}

The combat simulator under consideration here takes the general format as in Figure 1 (a), in which known inputs $\mathbf{X}$ are provided to the simulator, with known outputs $\mathbf{Y}$ captured as simulator metrics, repeated over a number of replications $R, r e p_{1}, \ldots, r e p_{R}$, used to reflect the stochastically-varying nature of the combat mission. The internal simulator data are recorded as the transition between event states $s_{1}, \ldots, s_{4}$ (Figure 1 (b)) yielding stochastically-varying temporally indexed event series, with these transitions necessarily varying for each replication $\mathrm{rep}_{1}, \mathrm{rep}_{2}, \ldots$. The features characterising the internal temporally indexed event space here include time of event, event type (Movement, Detection, Shots, Kills), spatial co-ordinates (latitude, longitude) and replication number $\mathrm{rep}_{j}$, representing which CRN value is used for the simulation.

Two competing approaches for this type of data are event time series, i.e. a set of events indexed by time, and event sequences, i.e. a set of times capturing a series of events. In this case study, events occur at inhomogeneous time points and are categorical, lending themselves to classical categorical time series models such as stochastic state-transition modelling (e.g. Markov chain models) and state-space models, such as categorical time series by treating the data as a discrete-valued time series (Weiss, 2018). A key challenge with these approaches lies in identifying an appropriate movement model in either continuous or discrete time; correlated random walks are used in the literature to capture state-switching behaviours, however complications arise when viewing the data as continuous-time versus as discrete instances of a continuous time process (Michelot \& Blackwell, 2019). A further challenge is that the simulated time index is irregular, requiring a time-irregularised first-difference correlated random walk model with drift, with an allowance for likely different autocorrelation levels in both spatial coordinates. Graph-based representations can be used with event sequences (Thejaswi, et al., 2020) although are typically used for pattern-detection problems in temporal graphs to determine usual or unusual event sequences rather than causal behaviour. One method that is data- 
driven and statistically robust to a number of these concerns, thus inherently designed to accommodate for all these design considerations, is Event Coincidence Analysis, discussed next.

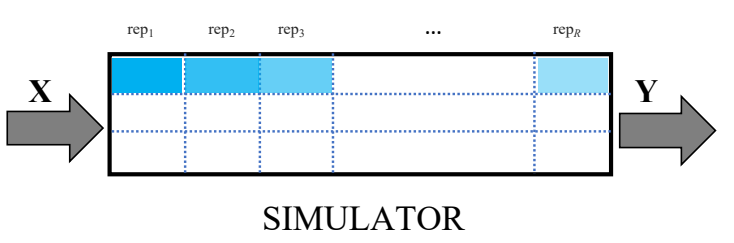

(a)

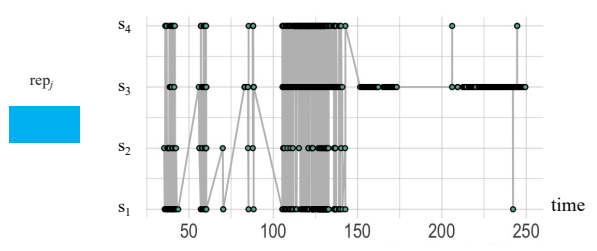

(b)

Figure 1. The CRN simulator structure in (a) with the internal black-box data indicated by the bolded rectangle; (b) internal black-box simulator transitions between event states $s_{1}, \ldots, s_{4}$ for replication $r e p_{j}$.

\section{MODELLING TEMPORALLY INDEXED EVENT SERIES USING ECA}

Event Coincidence Analysis captures causality between events (Donges, et al., 2016) by providing a framework for quantifying the strength, directionality (causality) and time lag between two binary event series. Moreover, ECA allows for significance testing of causality between two series, based on stochastic point processes with a prescribed inter-event time distribution and other higher-order properties (Donges, et al., 2016). Figure 2 illustrates ECA under two different premises; precursor and trigger causality event series. Specifically, Figure 2 (a) depicts the situation where an event in Series B acts as a precursor to an event in series A, where events of type B are typically observed before an event of type A takes place, but are not directly responsible for event A itself. Conversely, Figure 2 (b) represents the situation where an event of type B acts as a direct trigger for an event of type $\mathrm{A}$ and is considered responsible for event $\mathrm{A}$ occurring. In either case, time parameters $\Delta T$ and $\tau$ are required to determine whether a coincidence has occurred. An instantaneous coincidence occurs if two events with timings $t_{j}^{B}<t_{i}^{A}$ are closer in time than a coincidence interval $\Delta T$, allowing the option to address uncertain timings of events. A lagged coincidence $\tau$ is also incorporated to yield the coincidence window $\left(t_{i}^{A}-\tau\right)-t_{j}^{B} \leq \Delta T$ with $\tau \geq 0$ the lag parameter, to capture any natural lagging between the two event series. Thus ECA performs an asymmetric comparison between series to support directionality and allow for coincidence rate calculation.

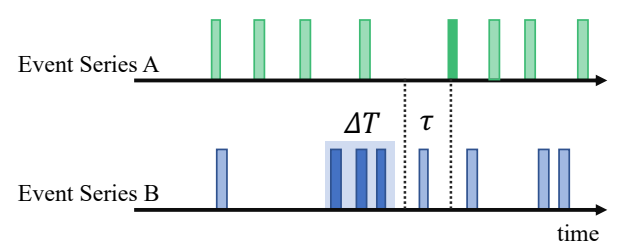

(a)

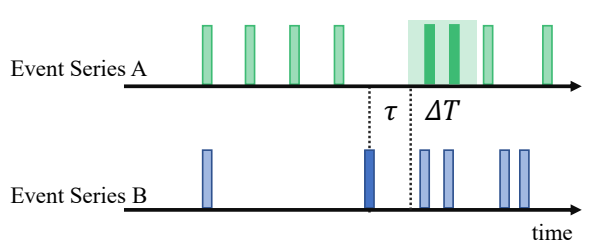

(b)

Figure 2. ECA for (a) a precursor event and (b) a trigger event. Event Series B causes Event Series A, with dark blue (green) bars indicating significant causal events. Shaded regions demarcate a significant coincidence window $\left(t_{i}^{A}-\tau\right)-t_{j}^{B} \leq \Delta T$. Adapted from (Donges, et al., 2016).

The calculations for the precursor coincidence rate $r_{p}(\Delta T, \tau)$ (Equation 1) and the equivalent for the trigger coincidence rate $r_{t}(\Delta T, \tau)$ (Equation 2$)$ are:

$r_{p}(\Delta T, \tau)=\frac{1}{N_{A}} \sum_{i=1}^{N_{A}} \Theta\left(\sum_{j=1}^{N_{B}} \mathbf{1}_{[0, \Delta T]}\left(\left(t_{i}^{A}-\tau\right)-t_{j}^{B}\right)\right)$

$r_{t}(\Delta T, \tau)=\frac{1}{N_{B}} \sum_{j=1}^{N_{B}} \Theta\left(\sum_{i=1}^{N_{A}} \mathbf{1}_{[0, \Delta T]}\left(\left(t_{i}^{A}-\tau\right)-t_{j}^{B}\right)\right)$

Here $N_{A}$ and $N_{B}$ are the number of events in series $\mathrm{A}$ and $\mathrm{B},\left\{t_{i}^{A}\right\}_{i=1}^{N_{A}},\left\{t_{j}^{B}\right\}_{j=1}^{N_{B}}$ the timings of events in series $\mathrm{A}$ and $\mathrm{B}$ respectively, $\Theta(\cdot)$ is the Heaviside unit step function ( 0 for $x \leq 0,1$ for $x>0)$ and $\mathbf{1}_{[0, \Delta T]}$ is an indictor function over the interval $[0, \Delta T]$ (equal to 1 for $x \in[0, \Delta T]$ and 0 otherwise). Significance testing for ECA allows for different possibilities depending on the statistical properties of the data - here we adopted a 
Poissonian approximation requiring events $\mathrm{A}$ and $\mathrm{B}$ to be randomly distributed and sufficiently rare $(\Delta T \ll$ $T / N_{A} \ll T$ ) where $T$ is the length of the binary sequence (Donges, et al., 2016).

Extensions of ECA include aggregation and conditionalisation; the former providing an integrated measure for coincidences that occur between several pairs of event series subject to some meaningful grouping mechanism and the latter allowing for the flexibility of interlinking multiple causal event series, to allow for conditioning of events on specific situations. Aggregated ECA has been used in the literature for spatiotemporal aggregation across different regions or countries for analysis of flooding and epidemic outbreaks (Donges, et al., 2016) while conditionalisation has appeared in the context of meteorological drivers of climatic extremes (Siegmund, et al., 2016). Aggregated precursor and trigger coincidence rates $r_{p}^{G}$ and $r_{t}^{G}$ for a group $G$ are given by Equations (3) and (4) while the conditionalised counterparts are provided in Equations (5) and (6).

$$
\begin{aligned}
& r_{p}^{G}(\Delta T, \tau)=\frac{\sum_{k \in G} \sum_{i=1}^{N_{A}, k} \Theta\left(\sum_{j=1}^{N_{B, k}} \mathbf{1}_{[0, \Delta T]}\left(\left(t_{i}^{A, k}-\tau\right)-t_{j}^{B, k}\right)\right)}{\sum_{k \in G} N_{A, k}} \\
& r_{t}^{G}(\Delta T, \tau)=\frac{\sum_{k \in G} \sum_{i=1}^{N_{B}, k} \Theta\left(\sum_{j=1}^{N_{A, k}} \mathbf{1}_{[0, \Delta T]}\left(\left(t_{i}^{A, k}-\tau\right)-t_{j}^{B, k}\right)\right)}{\sum_{k \in G} N_{B, k}} \\
& r_{c p}\left(\Delta T, \tau, \Delta T_{\text {cond }}, \tau_{\text {cond }}\right)=\frac{1}{N_{A}} \sum_{i=1}^{N_{A}} \Theta\left(\sum_{j=1}^{N_{B}} \Theta\left(\sum_{k=1}^{N_{C}} \mathbf{1}_{\left[0, \Delta T_{\text {cond }}\right]}\left(\left(t_{j}^{B}-\tau_{\text {cond }}\right)-t_{k}^{C}\right)\right) \mathbf{1}_{[0, \Delta T]}\left(\left(t_{i}^{A}-\tau\right)-t_{j}^{B}\right)\right) \\
& r_{c t}\left(\Delta T, \tau, \Delta T_{\text {cond }}, \tau_{\text {cond }}\right)=\frac{1}{N_{B}, \text { cond }} \sum_{j=1}^{N_{B}} \Theta\left(\sum_{i=1}^{N_{A}} \Theta\left(\sum_{k=1}^{N_{C}} \mathbf{1}_{\left[0, \Delta T_{\text {cond }}\right]}\left(\left(t_{j}^{B}-\tau_{\text {cond }}\right)-t_{k}^{C}\right)\right) \mathbf{1}_{[0, \Delta T]}\left(\left(t_{i}^{A}-\tau\right)-t_{j}^{B}\right)\right)
\end{aligned}
$$

Here $N_{C}$ is the number of events in series $\mathrm{C},\left\{t_{k}^{C}\right\}_{k=1}^{N_{C}}$ the timings of these events, $\Delta T_{\text {cond }}$ is a tolerance window for the conditional series, $\tau_{\text {cond }}$ is a time lag parameter for the conditional series and $N_{B}$, cond is the number of conditional events of type B having significant precursor coincidence with at least one event of type C.

It should be noted that aggregated coincidence rates support systematic analysis of coincidences under a grouping mechanism. Recalling the CRN combat simulator design (Figure 1), aggregation could thus occur over input or output metrics or anomalous simulations. In what follows we will focus on the latter, using aggregation to construct a combat narrative for those event sequences from anomalous simulations.

\section{CONSTRUCTING A COMBAT NARRATIVE USING ECA}

In this section we explore the use of ECA, using the R library coincalc (Siegmund, 2017), to construct a combat narrative. A key requirement of ECA is that data from the original observation space must be binarised with 0 indicating no event and 1 indicating an event has taken place. In this case study four event series - Movement, Detections, Shots and Kills - were binarised by identifying a subset of actions as events. Specifically, the event for Movement was standing, Detection was being identified or recognised, Shots was being hit and Kills was a catastrophic kill or mounted personnel casualty. Thus in what follows, combat narratives centre around the progression of Movement leading to Detection, being Shot and a Kill.

The first pair in this narrative is Movement causing Detection. The simulated movement event series was selected from the timepoint at which standing was first recorded and the Detection series was taken from the first timepoint at which identification or recognition occurred after the first standing event, to preserve the directionality of causal events. It was not possible to select identical time points as the events were time indexed, rather than recorded at regularly sampled intervals. To support the requirement that events $\mathrm{A}$ and $\mathrm{B}$ are sufficiently rare $\left(\Delta T \ll T / N_{A}\right)$, the parameter $\Delta T$ was set to 1 , thereby necessitating $\tau=0$, which tests whether an event of Type A was triggered by the closest event of Type B. This selection was supported by the time indices recorded within the simulator reflecting quick action during combat. A total of 200 temporally indexed events were considered, capturing a short time window over which most of the simulated events took place. The rare events condition was met, allowing for the Poissonian approximation significance test of the coincidence rates.

A subset of the Movement-Detection series is given in Figure 3 (a) with $r_{p}=0.819(p<.000)$ and $r_{t}=$ $0.59(p<.000)$ for the full-length sequences. These rates suggest a combat narrative that standing can act as either a precursor or trigger to being identified or recognised. The subsequent causal series (Detection-Shots, Figure $3(\mathrm{~b}))$ yielded $r_{p}=0.847(p=.008)$ and $r_{t}=0.381(p>.05)$. Here the combat narrative suggests it is a collection of precursory behaviours that lead to being shot after detection, rather than being shot as an immediate consequence of being identified or recognised. It is interesting to note $r_{p}=0.661(p=.06)$ and $r_{t}=0.245(p=.697)$ are both not significant for Movement-Shots, indicating the intervening Detection event is a necessary part of the causal chain of behaviour. 
To further investigate the mediated relationship Movement-Detection-Shot, conditionalised ECA was employed with $\Delta T=0, \tau=1, \Delta T_{\text {cond }}=0$ and $\tau_{\text {cond }}=1$ with these parameters also set to reflect relatively quick action during combat as captured by the time indices. Both the precursor and trigger coincidence rates were significant; $r_{c p}=0.566(p=.001)$ and $r_{c t}=0.408(p=.01)$ with conditionalised causality as depicted in Figure 4 (a) and (b).
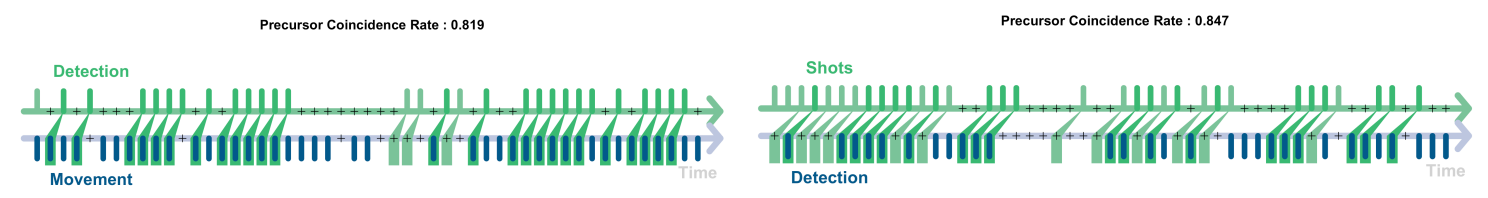

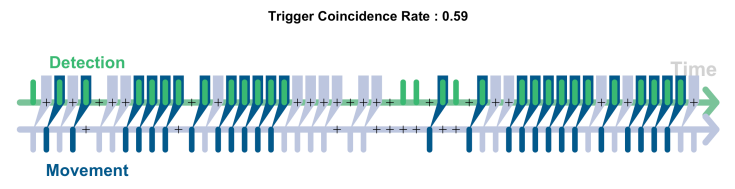

(a)

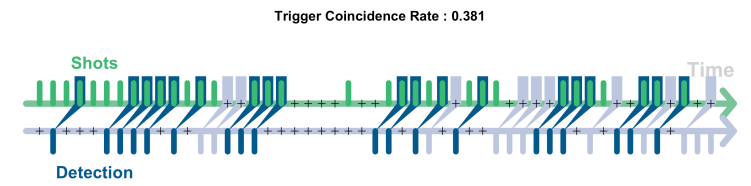

(b)

Figure 3 - A subset of the full sequence of (a) Movement (blue) causing Detection (green) and (b) Detection (blue) causing a Shot (green). Overlayed dark blue/green show significant coincidences (causal events).

From Figure 4 (a) and (b) it can be seen the combat narrative is filtered into a smaller set of coincidental events. That is, while there are a large number of significant Movement-Detection events, the number of significant Detection-Shots events is considerably smaller, indicating that being detected does not necessarily lead to being shot. For precursor coincidences there are 9 significant Detection-Shots events (Figure 4 (a)), suggesting that a minority of precursor behaviours can lead to the sequence of events Movement-Detections-Shots. This filtering effect is somewhat less stringent for trigger behaviour (Figure 4 (b)) with 15 significant DetectionShots events, suggesting there are more instances of a chains of events leading to being shot as a direct cause of detection.

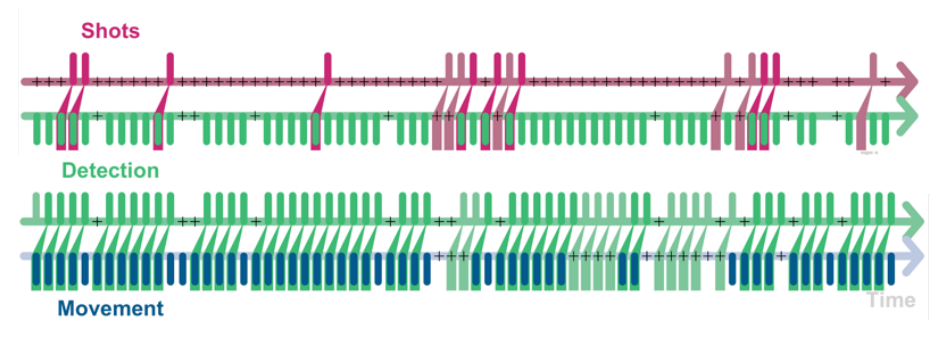

(a)

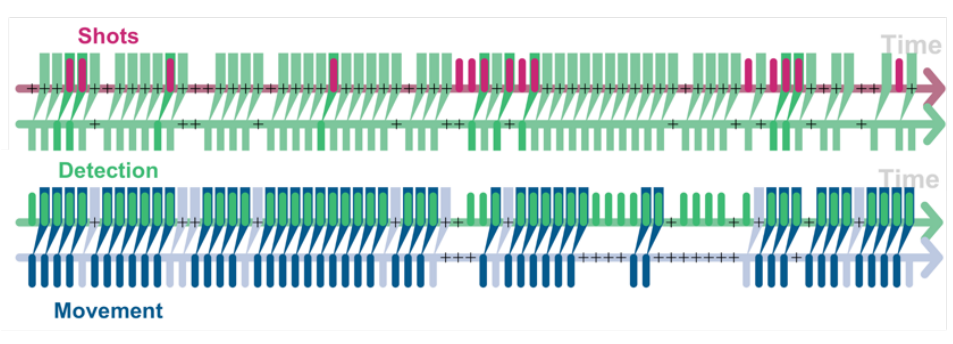

(b)

Figure 4 - (a) Precursor coincidences and (b) Trigger coincidences for conditional ECA. Overlayed dark blue/green/pink bars show significant coincidences (causal events).

The final investigation considered here used aggregated ECA to characterise anomalous behaviour as part of a combat narrative. Previous research (Schultz, et al., 2020) identified 36 anomalous replications in the event series Detections, Shots and Kills, with 16 of these replications resulting in mission failure. While mission failure is typically a function of the output produced by the simulator, it is unclear which of the intermediate 
event series, if any, may have contributed to this eventual outcome. A combat narrative could therefore provide invaluable insight into understanding why the mission was not classified as successful.

The three series were considered in two progressive steps; Detection as a precursor or trigger for Shots, followed by Shots as a precursor or trigger for Kills, with $\Delta T=0$ and $\tau=1$ in both cases. A total of 9 of 16 anomalous replications for Detections-Shots yielded significant precursor coincidence rates ranging between $0.592-0.754$ while only 2 replications yielded significant precursor rates in the subsequent Shots-Kills event series, with rates ranging between $0.818-0.820$. Interestingly, the Detection-Shots-Kills trio displayed continuity for 1 simulated replication only $\left(r_{p}=0.652(p=.006)\right.$ and $r_{p}=0.818(p=.022)$ for DetectionShots and Shots-Kills respectively), whereas the second significant Shots-Kills event did not yield a significant precursor event for Detection-Shots $\left(r_{p}=0.338(p=.980)\right)$.

A very different result was obtained for trigger coincidences; for Detection-Shots only 4 of 16 replications yielded significant coincidence rates ranging between $0.421-0.699$. However, the event sequence Shots-Kills showed no trigger coincidence rates were significant, suggesting that within the subset of anomalous scenarios that resulted in mission failure, a common feature is that none of the shots during the ultimately failed mission led to a kill event. Thus the suggested overarching combat narrative for these anomalous simulations indicate it is precursor behaviour that leads to being shot and potentially killed, rather than trigger behaviour, for those missions which are overall deemed as a failure.

At this point it is noted there are several limitations of the study presented here. The first is the choice of events for binarisation; further investigation is needed to enrichen the library of potentially differing narratives that may arise as a result of shifting focus from one subset of events to another subset of interest. The second is a detailed study on the choice of $\Delta T, \tau$ and the impact on the uncovered narratives. The third is that the results presented here are expected, in that the ECA-supported combat narrative is unsurprising. As a first step in this relatively new field of exploration the results presented here are encouraging in that more obvious narratives are supported by the methodology, however of stronger interest for future work would be uncovering those narratives which are not intuitive and, for example, would lend insight into mission outcomes that were unexpected. This would necessarily need to be supported by more detailed and broader simulation data to include numerous events for consideration. However in spite of these limitation, the first step towards constructing a combat narrative, based on simulated stochastically-varying temporally indexed events has been encouraging. It appears the use of Event Coincidence Analysis in its original, conditionalised and aggregated forms warrants further exploration for adaptation and applicability in this area to support decision-making using combat simulators.

\section{CONCLUSION}

In this paper we presented a first step towards constructing a causality-driven combat narrative via Event Coincidence Analysis, in support of decision-making when faced with competing simulator input design points. We considered a black-box combat simulator capturing stochastically-varying temporally indexed events Movement, Detection, Shots and Kills, to determine whether causality interlinked these sequences under typical and anomalous operating conditions.

Three different approaches for ECA were trialled. The first was paired events capturing the progression between the sequences Movement-Detection and Detection-Shots, for which a suggested combat narrative was that standing can act as either a precursor or trigger to being identified or recognised. Detection itself was not a trigger for being shot, instead playing a key mediating role between initial movement a shot being fired.

Conditional ECA further refined this narrative by filtering the Movement-Detection-Shot events into a smaller subset of causal behaviours to support the original narrative that Movement acts as a precursor to Detection but does not necessarily lead to being shot as a direct consequence. In terms of precursory behaviour, the narrative indicated that Movement and Detection were necessary for a shot to occur.

We also considered aggregated ECA for anomalous combat sequences that led to mission failure. A combat narrative was sought in terms of the interaction between Detection, Shots and Kills for these missions, with results indicating that for anomalous simulations precursor behaviour leads to being shot and potentially killed, rather than behaviour that directly triggers being shot and perhaps contributing to an unsuccessful mission. 
Future work will explore the choice of $\Delta T, \tau$ and their impact on the combat narrative including investigating longer lead times to precursor and/or trigger events. An expanded definition of events in each of the temporal sequences also will be investigated over a statistically sympathetic structure such as a network including coincident network structures, avoiding the need for selecting the time-based parameters $\Delta T$, $\tau$, or Markov logic networks, to allow for probabilistic transitions between events resulting in a predictive capability within the combat narrative thereby enhancing the reactive approach presented here. Other extensions include combat team labelling to identify team members, combined with graph theoretic methods such as social network analysis, to determine key players and their roles to further enrichen the combat narrative.

\section{ACKNOWLEDGMENTS}

The Commonwealth of Australia (represented by the Defence Science and Technology Group) supported this research through a Defence Science Partnership agreement.

We would like to thank Dr Andrew Gill for his invaluable input into this research through expert insight. We would also like to thank the anonymous reviewers for their insightful comments and helpful suggestions.

\section{REFERENCES}

Donges, J. F., Schleussner, C.-F., Siegmund, J. F. \& Donner, R. V., 2016. Event coincidence analysis for quantifying statistical interrelationships between event time series. Eur. Phys. J. Spec. Top., 225(3), p. 471487.

Fdez-Arroyabe, P. et al., 2020. Schumann resonance and cardiovascular hospital admission in the area of Granada, Spain: An event coincidence analysis approach. Science of the Total Environment, Volume 705:135813.

Heikes, R. G., Montgomery, D. C. \& Rardin, R. L., 1976. Using common random numbers in simulation experiments — an approach to statistical analysis. SIMULATION, 27(3), p. 81—85.

Law, A., 2015. Simulation Modeling and Analysis. 5th edn ed. New York, USA: McGraw-Hill.

Michelot, T. \& Blackwell, P. G., 2019. State-switching continuous-time correlated random walks. Methods in Ecology and Evolution, Volume 10, pp. 637-649.

Scharwaechter, E. \& Mueller, E., 2020. Does terrorism trigger online hate speech? On the association of events and time series. The Annals of Applied Statistics, 14(3), pp. 1285-1303.

Schleussner, C.-F., Donges, J. F., Donner, R. V. \& Schellnhuber, H. J., 2016. Armed-conflict risks enhanced by climate-related disasters in ethnically fractionalized countries. PNAS, 113(33), pp. 9216-9221.

Schultz, L., Chiera, B. \& John, M., 2020. Simulation Pattern Recognition and Outlier Classification for Key Events Tool (SPROCKET) - Phase 2. Adelaide: DSTG.

Siegmund, J., 2017. CoinCalc. [Online] Available at: https://github.com/JonatanSiegmund/CoinCalc [Accessed 0107 2021].

Siegmund, J. F. et al., 2016. Meteorological Drivers of Extremes in Daily Stem Radius Variations of Beech, Oak, and Pine in Northeastern Germany: An Event Coincidence Analysis. Frontiers in Plant Science, Volume 7, p. 14.

Templ, B. et al., 2021. Coincidence of temperature extremes and phenological events of grapevines. OENO One, 55(1), p. 367-383.

Thejaswi, S., Gionis, A. \& Lauri, J., 2020. Finding path motifs in large temporal graphs using algebraic fingerprints. Big Data, 8(5), p. 335-362.

Weiss, C. H., 2018. An introduction to discrete-valued time series. 1st ed. Hoboken, New Jersey: Wiley.

Wiedermann, M., Siegmund, J. F., Donges, J. F. \& Donner, R. V., 2021. Differential Imprints of Distinct ENSO Flavors in Global Patterns of Very Low and High Seasonal Precipitation. Frontiers in Climate, 3(Article 618548), p. 5.

Wolf, F., Ozturk, U., Cheung, K. \& Donner, R. V., 2021. Spatiotemporal patterns of synchronous heavy rainfall events in East Asia during the Baiu season. Earth System Dynamics, 12(1), pp. 295-312. 